\title{
Combined Effect of Pressure and Shear Stress on Penny- Shaped Fluid-Driven Cracks
}

Chinese Academy of Sciences,

Beijing 100190, China;

School of Engineering Science, University of Chinese Academy of Sciences, Beijing 100049, China

Ya-Pu Zhao ${ }^{1}$

State Key Laboratory of

Nonlinear Mechanics (LNM), Institute of Mechanics,

Chinese Academy of Sciences, Beijing 100190, China;

School of Engineering Science, University of Chinese Academy of Sciences, Beijing 100049, China e-mail: yzhao@imech.ac.cn
Penny-shaped fluid-driven cracks are often detected in many fluid-solid interaction problems. We study the combined effect of pressure and shear stress on the crack propagation in an impermeable elastic full space. Boundary integral equations are presented, by using the integral transform method, for a penny-shaped crack under normal and shear stresses. The crack propagation criterion of stress intensity factor is examined with the strain energy release rate. Dominant regimes are obtained by using a scaling analysis. Asymptotic solution of the toughness-dominant regime is derived to show the effect of shear stress on the crack opening, crack length, and pressure distribution. The results indicate that a singular shear stress can dominate the asymptotic property of the stress field near the crack tip, and the stress intensity factor cannot be calculated even though the energy release rate is finite. Shear stress leads to a smaller crack opening, a longer crack, and a slightly larger wellbore pressure. A novel dominant-regime transition between shear stress and pressure is found. Unstable crack propagation occurs in the shear stress-dominant regime. This study may help in understanding crack problems under symmetrical loads and modeling fluid-solid interactions at the crack surfaces. [DOI: $10.1115 / 1.4038719]$

Keywords: penny-shaped cracks, shear stress, crack propagation criterion, fluid-driven cracks

\section{Introduction}

Fluid-driven cracks under symmetrical pressure and shear stress on the crack surfaces are observed in various fluid-solid interaction problems, such as magma (or water) driven cracks in the earth crust (or glacier beds) [1-3], the fabrication of flexible structures and electronics [4], the storage of carbon dioxide under the ground $[5,6]$, and hydraulic fracturing in the oil and gas industry to enhance the production [7-15]. In the problems, a viscous fluid flows into the channel between the crack surfaces generating symmetrical normal and shear stresses on the crack surfaces and further forcing the solid to be fractured gradually. The problems are pure mode-I because both the normal and shear stresses are symmetrical about the crack plane.

Most of the existing models assume that the shear stress is negligible compared to the normal stress $[1-4,7,8,11,12]$; however, recent studies revealed that a singular shear stress may cause a singular stress field with a square-root singularity, which is of the same order as that caused by a normal stress $[9,10]$. This shear stress-induced stress-singularity problem is due to the lack of physical details near the crack tip. Physically, there is a fluid-lag zone between the fluid tip and the crack tip [11]. The fluid-lag zone is small and often neglected in modeling the problem. At the crack tip, the lack of this physical detail leads to singular normal and shear stresses acting on the crack surfaces because of the use of lubrication theory approximation $[9,12]$. The asymptotic properties of displacement and stress fields change near the crack tip.

The change in the asymptotic properties of stress fields results in the requirement of the examination of the crack propagating criteria. One of the most often used criteria is the stress intensity factor being equal to the fracture toughness. The calculation of stress intensity factor relies on the square-root singularity of stress field at the crack tip. However, a stronger singularity of stress field may be obtained due to the shear stress [10]. Thus, the criterion of

\footnotetext{
${ }^{1}$ Corresponding author.

Manuscript received October 12, 2017; final manuscript received December 7 , 2017; published online January 4, 2018. Assoc. Editor: N. R. Aluru.
}

stress intensity factor requires an examination for hydraulic fracturing. There are other criteria for mode-I cracks such as strain energy release rate and $J$-integral. Only the strain energy release rate is discussed because of the equivalence of the two criteria for linear elastic materials. The strain energy release rate is calculated as the validation of the stress intensity factor $[16,17]$. Owing to the existence of shear stress acting on the crack surfaces, the conclusion that zero-stress intensity factor results in zero-energy release rate may be conditionally right.

Unstable crack propagation was reported for pure mode-I plane-strain fluid-driven cracks [10]. A negative shear stress acting on the upper crack surface, which is positive on the lower crack surface, tends to inhibit the crack. In the viscosity-dominant regime, the crack surfaces overlap near the crack tip under the shear stress, indicating an unstable crack propagation. Singular stress intensity factor and energy release rate were obtained. Either criteria are inappropriate under the assumption that the crack is fractured straightly and steadily without a fluid lag. For penny-shaped fluid-driven cracks that are subjected to different scaling laws of crack opening, crack length and pressure [18,19], the combined effect of symmetrical normal and shear stresses on the stress intensity factor and strain energy release rate remains unsolved.

In this paper, the combined effect of pressure and shear stress on fluid-driven penny-shaped cracks is analyzed. The fracturing fluid is assumed of power-law rheology and modeled by lubrication theory. The theory of linear elastic fracture mechanics is used to describe the deformation and fracture behavior of a brittle solid. By using the integral transform method $[4,20]$, the boundary integral equations of displacement and stress fields are derived. And the shear stress-dependent stress intensity factor is derived from the stress filed in front of the crack tip. In the toughness-dominant regime, the crack-propagation behavior with the shear stressdependent stress intensity factor is analyzed by deriving an asymptotic solution. The crack propagation criterion based on stress intensity factor is examined with the energy release rate. Near-crack-tip asymptotics are used in the viscosity-dominant regime where stress intensity factor may be singular. It is found that there can be instabilities and failures at the crack center, and 
the dominant-regime transition from shear stress to pressure is discovered.

\section{Mathematical Expression}

A penny-shaped crack of length $l$ is in the linear-elastic full space, as shown in Fig. 1. The cylindrical coordinate system is used such that the origin coincides with the crack center and the $r$ axis is parallel to, and the $z$-axis is perpendicular to, the crack plane. There are symmetrical normal and shear stresses $p$ and $\tau$ acting on the crack surfaces and uniform compressive stress, $\sigma_{\infty}$, at infinity. For convenience and without loss of generality, we focus on the first quadrant where $r>0$ and $z>0$.

2.1 Fluid Flow. Lubrication theory is used to model the fracturing fluid with power-law rheology. Local mass conservation gives

$$
\frac{\partial w(r, t)}{\partial t}+\frac{1}{2 \pi r} \frac{\partial q(r, t)}{\partial r}=0
$$

where $w(r, t)$ and $q(r, t)$ are the distribution of crack opening and flow rate, respectively. The relation between the flow rate, $q(r, t)$, and pressure, $p(r, t)$, is given by

$q(r, t)=-\operatorname{sgn}\left\{\frac{\partial p(r, t)}{\partial r}\right\} \frac{4 n \pi r}{C^{1 / n} 2^{(2 n+1) / n}(2 n+1)}\left|\frac{\partial p(r, t)}{\partial r} w^{2 n+1}(r, t)\right|^{\frac{1}{n}}$

where $\operatorname{sgn}\{*\}$ is the Signum function, $C$ and $n$ are the flow consistency index and flow behavior index, respectively. A detailed derivation of Eqs. (1) and (2) is given in Appendix A. Flow rate is pregiven at the wellbore, $r=0$, and at the crack tip, $r=l(t)$, as

$$
q(0, t)=q_{0} \quad \text { and } \quad q(l(t), t)=0
$$

Physically, there is a wellbore and a fluid-lag zone at the center and the tip of the crack, respectively. For a long crack, the radius of the wellbore can be neglected [11]. It is known from Ref. [13] that the fraction of the fluid-lag zone to the half-length of the crack decreases quickly during the hydraulic fracturing under the action of crustal stress, and the effect of the fluid-lag zone can be neglected at a large time. Consequently, the details of the center and the tip of the crack are assumed to be negligible. Global mass conservation gives

$$
q_{0} t=2 \pi \int_{0}^{l(t)} w(\zeta, t) \zeta \mathrm{d} \zeta
$$

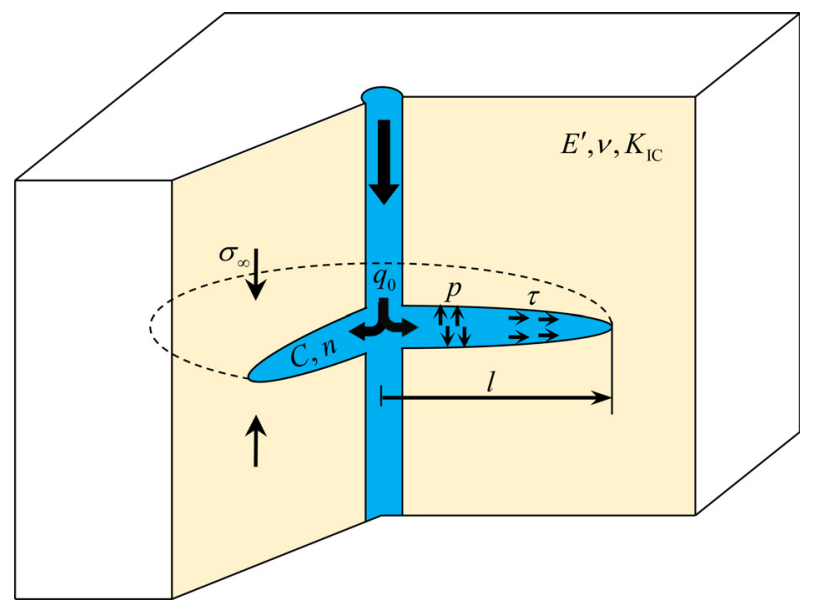

Fig. 1 A penny-shaped crack driven by a viscous fluid flow and a uniform normal stress at infinity which is dependent on Eq. (3) $)_{1}$. Here, Eq. (4) is used for convenience. The relationship between pressure and the crack opening is obtained, by integrating Eq. (1) with the boundary condition Eq. (3) $)_{2}$ and substituting the result into Eq. (2), as

$$
\frac{\partial p(r, t)}{\partial r}=-\operatorname{sgn}\left\{\int_{r}^{l(t)} \frac{\partial w(\zeta, t)}{\partial t} \zeta \mathrm{d} \zeta\right\} \frac{C^{\prime}}{w^{2 n+1}(r, t)}\left|\frac{1}{r} \int_{r}^{l(t)} \frac{\partial w(\zeta, t)}{\partial t} \zeta \mathrm{d} \zeta\right|^{n}
$$

in which $C^{\prime}=C \times 2^{2 n+1}[(2 n+1) / 2 n]^{n}$. The shear stress can be obtained by force equilibrium as

$$
\tau(r, t)=\frac{w(r, t)}{2} \frac{\partial p(r, t)}{\partial r}
$$

2.2 Quasi-Static Crack Propagation. By using the Hooke's law, $\boldsymbol{\sigma}=\lambda \gamma \mathbf{I}+2 \mu \gamma$, the equations of equilibrium are [4]

$$
\begin{gathered}
\nabla^{2} \boldsymbol{u}+\frac{1}{1-2 \nu} \nabla \gamma-\frac{\boldsymbol{u}}{r^{2}}=0 \\
\nabla^{2} \gamma=0
\end{gathered}
$$

where $\gamma=\nabla \cdot \boldsymbol{u}$ is the dilatation of an infinitesimal element, $\nu$ is the Poisson's ratio, $\nabla=\boldsymbol{e}_{r}(\partial / \partial r+1 / r)+\boldsymbol{e}_{z} \partial / \partial z$ and $\nabla^{2}=\partial^{2} / \partial r^{2}+(1 / r) \partial / \partial r+\partial^{2} / \partial z^{2}$.

The far-field stress can be decomposed with the superposition method. The assumption of $\sigma_{\infty}=0$ is used in the following for convenience. The dynamic conditions on the crack surface of the original crack problem are:

$$
\begin{gathered}
\sigma_{r z}\left(r, 0^{+}\right)= \begin{cases}\tau(r), & r \in(0, l) \\
0, & r \in(l, \infty)\end{cases} \\
\sigma_{z z}\left(r, 0^{+}\right)=-p(r), \quad r \in(0, l)
\end{gathered}
$$

and the related kinematic boundary condition is

$$
u_{z}\left(r, 0^{+}\right)=0, \quad r \in(l, \infty)
$$

The normal displacement is coupled with the crack opening as

$$
u_{z}\left(r, 0^{+}\right)-u_{z}\left(r, 0^{-}\right)=2 u_{z}\left(r, 0^{+}\right)=w
$$

2.3 Solution to the Crack Problem. Hankel transforms are used to solve Eqs. (7) and (8) with the boundary-condition equations (9)-(11) [10,20]. The displacement and stress components are, therefore

$u_{r}(r, z)=-\frac{1}{\pi \mu}\left[(1-2 \nu) I_{\mathrm{P} 10}+2(1-\nu) I_{\mathrm{T} 10}\right]+\frac{1}{\pi \mu}\left(z I_{\mathrm{P} 11}+z I_{\mathrm{T} 11}\right)$

$$
u_{z}(r, z)=\frac{1}{\pi \mu}\left[2(1-\nu) I_{\mathrm{P} 00}+(1-2 \nu) I_{\mathrm{T} 00}\right]+\frac{1}{\pi \mu}\left(z I_{\mathrm{P} 01}+z I_{\mathrm{T} 01}\right)
$$

$$
\begin{aligned}
\sigma_{r r}(r, z)=- & \frac{2}{\pi}\left(I_{\mathrm{P} 01}+2 I_{\mathrm{T} 01}\right)+\frac{2}{\pi}\left(z I_{\mathrm{P} 02}+z I_{\mathrm{T} 02}\right) \\
& +\frac{2}{\pi}\left[(1-2 \nu) \frac{1}{r} I_{\mathrm{P} 10}+2(1-\nu) \frac{1}{r} I_{\mathrm{T} 10}\right] \\
& -\frac{2}{\pi}\left(\frac{z}{r} I_{\mathrm{P} 11}+\frac{z}{r} I_{\mathrm{T} 11}\right)
\end{aligned}
$$




$$
\begin{gathered}
\sigma_{r z}(r, z)=\frac{2}{\pi} I_{\mathrm{T} 11}-\frac{2}{\pi}\left(z I_{\mathrm{P} 12}+z I_{\mathrm{T} 12}\right) \\
\sigma_{z z}(r, z)=-\frac{2}{\pi} I_{\mathrm{P} 01}-\frac{2}{\pi}\left(z I_{\mathrm{P} 02}+z I_{\mathrm{T} 02}\right)
\end{gathered}
$$

where $I_{\mathrm{P} i j}$ and $I_{\mathrm{T} i j}$ are defined by

$$
\begin{aligned}
& I_{\mathrm{P} i j}(r, z)=\int_{0}^{l} \mathcal{I}_{i j}(\xi ; r, z) \mathrm{d} \xi \int_{0}^{\xi} \frac{\zeta p(\zeta) \mathrm{d} \zeta}{\sqrt{\xi^{2}-\zeta^{2}}} \\
& I_{\mathrm{T} i j}(r, z)=\int_{0}^{l} \mathcal{I}_{i j}(\xi ; r, z) \xi \mathrm{d} \xi \int_{\xi}^{l} \frac{\tau(\zeta) \mathrm{d} \zeta}{\sqrt{\zeta^{2}-\xi^{2}}}
\end{aligned}
$$

in which the subscripts " $P$ " and " $T$ " represent the normal and shear stresses, respectively, and $\mathcal{I}_{i j}$ is given in Table 1 . Thus, only the infinite integrals for $j=0$ are required to be solved. The solution for full space can be obtained, with the solution for the first quadrant and the mirror symmetry about the crack plane.

The calculation of displacement and stress on the crack surfaces and in front of the crack tip under symmetrical loads requires the integrations of $I_{\mathrm{P} 00}, I_{\mathrm{P} 10}, I_{\mathrm{P} 01}, I_{\mathrm{T} 00}, I_{\mathrm{T} 10}$, and $I_{\mathrm{T} 01}$, according to Eqs. (13)-(17). The solution based on iterated integrals is obtained by substituting Eqs. (18), (19), and $z=0$ into Eqs. (13)-(17). For $0<r<l$

$$
\begin{aligned}
u_{z}\left(r, 0^{+}\right)= & \frac{2(1-\nu)}{\pi \mu} \int_{r}^{l} \frac{\mathrm{d} \xi}{\sqrt{\xi^{2}-r^{2}}} \int_{0}^{\xi} \frac{\zeta p(\zeta) \mathrm{d} \zeta}{\sqrt{\xi^{2}-\zeta^{2}}} \\
& +\frac{1-2 \nu}{\pi \mu} \int_{r}^{l} \frac{\xi \mathrm{d} \xi}{\sqrt{\xi^{2}-r^{2}}} \int_{\xi}^{l} \frac{\tau(\zeta) \mathrm{d} \zeta}{\sqrt{\zeta^{2}-\xi^{2}}}
\end{aligned}
$$

\begin{tabular}{|c|c|c|c|}
\hline & $r>0, z>0$ & $z=0$ & $r=0, z>0$ \\
\hline $\mathcal{I}_{00}$ & $\operatorname{Im} \frac{1}{\sqrt{Z^{2}+r^{2}}}$ & $\frac{\mathbb{H}\{\xi-r\}}{\sqrt{\xi^{2}-r^{2}}}$ & $\frac{\xi}{z^{2}+\xi^{2}}$ \\
\hline $\mathcal{I}_{10}$ & $-\operatorname{Im} \frac{1}{r} \frac{Z}{\sqrt{Z^{2}+1}}$ & $\frac{\xi \mathbb{H}\{r-\xi\}}{r \sqrt{r^{2}-\xi^{2}}}$ & 0 \\
\hline $\mathcal{I}_{01}$ & & & $-\frac{\partial}{\partial z} \mathcal{I}_{00}$ \\
\hline $\mathcal{I}_{11}$ & & & 0 \\
\hline $\mathcal{I}_{02}$ & -( & $\mathcal{I}_{00}$ & $\frac{\partial^{2}}{\partial z^{2}} \mathcal{I}_{00}$ \\
\hline $\mathcal{I}_{12}$ & $-\left(\frac{\partial^{2}}{\partial r^{2}}\right.$ & $\left.\frac{1}{.2}\right) \mathcal{I}_{10}$ & 0 \\
\hline
\end{tabular}

and for $r>l$

$$
\begin{aligned}
\sigma_{r r}(r, 0)= & -\frac{2}{\pi r} \frac{\mathrm{d}}{\mathrm{d} r} \int_{0}^{l} \frac{\xi \mathrm{d} \xi}{\sqrt{r^{2}-\xi^{2}}} \int_{0}^{\xi} \frac{\zeta p(\zeta) \mathrm{d} \zeta}{\sqrt{\xi^{2}-\zeta^{2}}} \\
& -\frac{4}{\pi r} \frac{\mathrm{d}}{\mathrm{d} r} \int_{0}^{l} \frac{\xi^{2} \mathrm{~d} \xi}{\sqrt{r^{2}-\xi^{2}}} \int_{\xi}^{l} \frac{\tau(\zeta) \mathrm{d} \zeta}{\sqrt{\zeta^{2}-\xi^{2}}} \\
& +\frac{2(1-2 \nu)}{\pi r} \int_{0}^{l} \frac{\xi \mathrm{d} \xi}{r \sqrt{r^{2}-\xi^{2}}} \int_{0}^{\xi} \frac{\zeta p(\zeta) \mathrm{d} \zeta}{\sqrt{\xi^{2}-\zeta^{2}}} \\
& +\frac{4(1-\nu)}{\pi r} \int_{0}^{l} \frac{\xi^{2} \mathrm{~d} \xi}{r \sqrt{r^{2}-\xi^{2}}} \int_{\xi}^{l} \frac{\tau(\zeta) \mathrm{d} \zeta}{\sqrt{\zeta^{2}-\xi^{2}}}
\end{aligned}
$$

Table 1 The infinite integrals with $Z=\boldsymbol{Z}-\mathbf{i} \xi$ and $\mathbb{H}\{*\}$ being the Heaviside function

$$
\sigma_{z z}(r, 0)=-\frac{2}{\pi r} \frac{\mathrm{d}}{\mathrm{d} r} \int_{0}^{l} \frac{\xi \mathrm{d} \xi}{\sqrt{r^{2}-\xi^{2}}} \int_{0}^{\xi} \frac{\zeta p(\zeta) \mathrm{d} \zeta}{\sqrt{\xi^{2}-\zeta^{2}}}
$$

The iterated integrals can be reduced to single integrals, which are given in the Appendix B. By using Eq. (12), the crack opening can be calculated with

$$
w(r, t)=\frac{8}{\pi E^{\prime}} \int_{0}^{l(t)} K_{\mathrm{P} 00}(\zeta ; r) p(\zeta, t) \mathrm{d} \zeta+\frac{2(1-2 \nu)}{(1-\nu) E^{\prime}} \int_{r}^{l(t)} \tau(\zeta, t) \mathrm{d} \zeta
$$

where $E^{\prime}=2 \mu /(1-\nu)$ and $K_{\mathrm{P} 00}$ is defined in Appendix B.

Uniform loads acting on the crack surfaces are assumed to validate Eqs. (22) and (23) and to clearly show the effect of a negative shear stress. The analytical solution can be obtained for $z=0$ by integrating the normal stress of Eqs. (20) and (22) and the shear stress on the right-hand side of Eq. (B2) as

$$
\begin{gathered}
u_{z}\left(r, 0^{+}\right)=\frac{4}{\pi E^{\prime}} p_{0} \sqrt{l^{2}-r^{2}}+\frac{1-2 \nu}{(1-\nu) E^{\prime}} \tau_{0}(l-r), \quad r \in(0, l) \\
\sigma_{z z}(r, 0)=\frac{2}{\pi} p_{0}\left(\frac{l}{\sqrt{r^{2}-l^{2}}}-\operatorname{arccsc} \frac{r}{l}\right), \quad r \in(l, \infty)
\end{gathered}
$$

in which $p_{0}$ and $\tau_{0}$ are constant normal and shear stresses, respectively. Note that the uniform normal and shear stresses do not represent the pressure and shear stress in the fluid flow. For $\tau_{0}=0$, the solution degenerates to the formula of only a normal stress acting on the crack surfaces [20]. It is evident that a negative shear stress reduces the value of $u_{z}$, and leads to a non-zero first derivative of $u_{z}$ at the crack center, i.e., for a shear stress-only problem, the $u_{z}$ is negative and the upper and lower crack surfaces overlap each other. From the contour lines and streamlines of the displacement, as shown in Fig. 2, the material on the axis of symmetry moves toward the crack surface, and the material near the crack surfaces moves away from the crack center. According to the boundary conditions and property of axial symmetry, $\tilde{u}_{r}(0, \tilde{z})=0$ and $\tilde{u}_{z}\left(\tilde{r}, 0^{ \pm}\right)=0$ for $\tilde{r}>1$, the material has the ability to "slip" along the axes. The shear stress rotates the material counterclockwise for $\tilde{z}>0$ and clockwise for $\tilde{z}<0$. The rotation of the

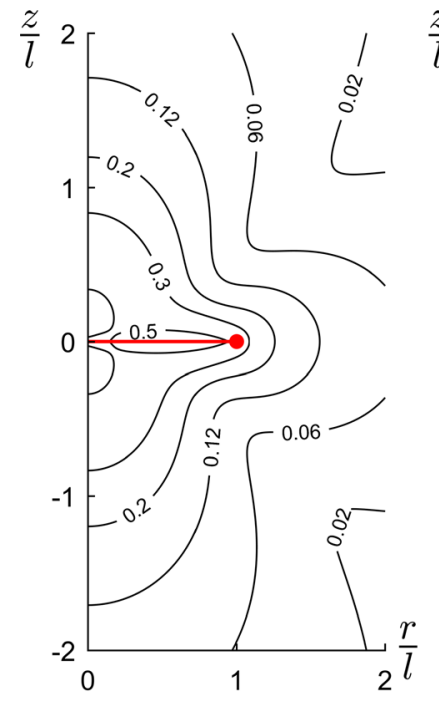

(a)

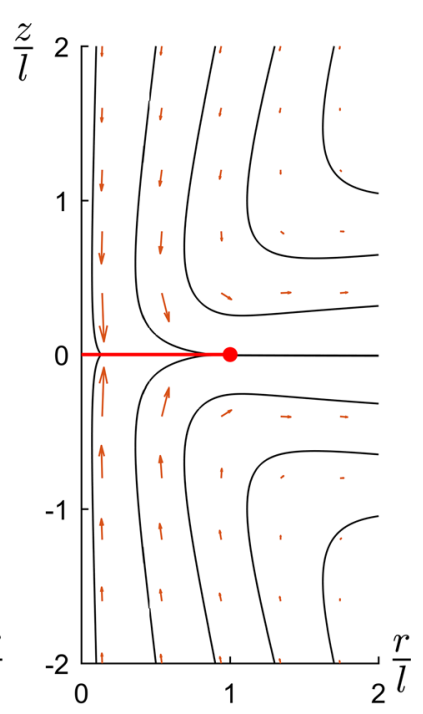

(b)
Fig. 2 Displacement field under uniform shear stress on the crack surfaces: (a) contour lines of total displacement and (b) streamlines of the displacement vector. The dot $(1,0)$ represents the crack tip 
material leads to the overlap of crack surfaces. Consequently, a negative shear stress tends to inhibit a crack.

\section{Shear Stress-Dependent Stress Intensity Factor and Energy Release Rate}

There are two kinds of energy dissipations, the fracturing of the rock and the viscous flow of the fluid, which have been fully studied [12-14,21] and reviewed by Detournay [19]. The toughnessdominant regime represents the work done by fracturing the rock being much more than that done by overcoming the friction of the fluid flow; and the viscosity-dominant regime is defined conversely. In the two different dominant regimes, the near-crack-tip asymptotics are different. In the toughness-dominant regime, the viscosity of the fracturing fluid is negligible, and the square-root singularity of the stress field at the crack tip holds. Whereas, in the viscosity-dominant regime, the fracture toughness is negligible, and the non-square-root singularity of the stress field at the crack tip can only be obtained with the coupling of fracture mechanics and lubrication theory. The stress intensity factor, $K_{\mathrm{I}}$, is commonly calculated as

$$
K_{\mathrm{I}}=\lim _{r \rightarrow l^{+}} \sqrt{2 \pi(r-l)} \sigma_{z z}(r, 0)=\frac{2}{\sqrt{\pi l}} \int_{0}^{l} \frac{\zeta p(\zeta) \mathrm{d} \zeta}{\sqrt{l^{2}-\zeta^{2}}}
$$

which is independent of the shear stress. Note that the hydraulic crack is modeled as pure mode-I because the pressure and shear stress are symmetrical about the $x_{1}$-axis. Here, the near-crack-tip properties of the boundary integral equations are studied and the crack propagation criteria of stress intensity factor and energy release rate are examined.

Near-crack-tip asymptotics are required to evaluate the stress intensity if the stress intensity factor is shear stress dependent. The asymptotic solutions of displacement and stress fields near the crack tip are known for the stress intensity factor being nonzero $[16,17]$. The hydraulic fracturing is assumed in the viscositydominant regime. Thus, the stress intensity factor is assumed to approach zero. It is known that the pressure and shear stress depend on the rheology of the fracturing fluid [19]. To overcome this difficulty, power-law loads are implemented separately for either a normal stress-only or a shear stress-only problem as

$$
p(r)=p_{0}\left[1-\left(1+2 e_{\mathrm{P}}\right)\left(1-\frac{r^{2}}{l^{2}}\right)^{e_{\mathrm{P}}}\right] \text { and } \tau(r)=\tau_{0}\left(1-\frac{r^{2}}{l^{2}}\right)^{e_{\mathrm{T}}}
$$

In which $e_{\mathrm{P}}$ and $e_{\mathrm{T}}$ are arbitrary constants subjected to $e_{\mathrm{P}}>-1 / 2$ and $e_{\mathrm{T}}>-1$, and $1+2 e_{\mathrm{P}}$ is used to vanish the $K_{\mathrm{I}}$. The minus sign in front of the parentheses of Eq. $(27)_{1}$ is from the fluid-solid interaction problem [9-12], where pressure decreases and shear stress is negative because of the fluid viscosity. According to a numerical calculation based on the Gauss-Chebyshev quadrature [22], there are

$$
\begin{array}{r}
u_{z}\left(r, 0^{+}\right)=A_{\mathrm{P}}\left(1-\frac{r^{2}}{l^{2}}\right)^{1+e_{\mathrm{P}}}+A_{\mathrm{T}}\left(1-\frac{r^{2}}{l^{2}}\right)^{1+e_{\mathrm{T}}} \\
+O\left[\left(1-\frac{r}{l}\right)^{3 / 2}\right]+O\left[\left(1-\frac{r}{l}\right)^{2+e_{\mathrm{T}}}\right] \\
\sigma_{r r}(r, 0)=B_{\mathrm{P}}\left(\frac{r^{2}}{l^{2}}-1\right)^{e_{\mathrm{P}}}+B_{\mathrm{T}}\left(\frac{r^{2}}{l^{2}}-1\right)^{e_{\mathrm{T}}}+O(1) \\
\sigma_{z z}(r, 0)=B_{\mathrm{P}}\left(\frac{r^{2}}{l^{2}}-1\right)^{e_{\mathrm{P}}}+O(1)
\end{array}
$$

where $A$ 's and $B$ 's are constants, $A_{\mathrm{P}}$ and $B_{\mathrm{P}}$ are positive for $e_{\mathrm{P}}$ being negative, and $A_{\mathrm{T}}$ and $B_{\mathrm{T}}$ are negative for a negative shear stress.

An improper choice of the stress component, to calculate the stress intensity factor, may lead to the exclusion of the effect of shear stress. From Eqs. (29) and (30), it is evident that stress intensity factor is conditionally influenced by shear stress. However, $\sigma_{r z}(r, 0)$ is zero for the pure mode-I crack and $\sigma_{z z}(r, 0)$ is independent of the shear stress according to Eq. (30). Thus, $\sigma_{r r}(r, 0)$ is used rather than $\sigma_{z z}(r, 0)$ to calculate $K_{\mathrm{I}}$ as $K_{\mathrm{I}}^{\prime}=\lim _{r \rightarrow l^{+}} \sqrt{2 \pi(r-l)} \sigma_{r r}(r, 0)$, where the prime symbol is used to distinguish from that calculated with $\sigma_{z z}(r, 0)$. From Eq. (29), there is

$$
K_{\mathrm{I}}^{\prime}=\frac{2}{\sqrt{\pi l}} \int_{0}^{l} \frac{\zeta p(\zeta) \mathrm{d} \zeta}{\sqrt{l^{2}-\zeta^{2}}}+\sqrt{\pi l} B_{\mathrm{T}} \lim _{r \rightarrow l^{+}}\left(\frac{r^{2}}{l^{2}}-1\right)^{e_{\mathrm{T}}+1 / 2}
$$

which is related to $K_{\mathrm{I}}$ as $K_{\mathrm{I}}^{\prime}=K_{\mathrm{I}}+\sqrt{\pi} B_{\mathrm{T}} \lim _{r \rightarrow l^{+}}\left(r^{2}-l^{2}\right)^{e_{\mathrm{T}}+1 / 2}$. A negative shear stress leads to a negative value of $B_{\mathrm{T}}$ and the decrease of the total stress intensity factor. This is in accord with the result of Sec. 2.3 that a negative shear stress tends to inhibit a crack. For $e_{\mathrm{T}}>-1 / 2, K_{\mathrm{I}}^{\prime}=K_{\mathrm{I}}$, the use of stress intensity factor is valid, and there is a square-root singularity of stress field at the crack tip. For $e_{\mathrm{T}}<-1 / 2, K_{\mathrm{I}}^{\prime}$ is infinite and the concept of stress intensity with a square-root stress singularity is inappropriate. For $e_{\mathrm{T}}=-1 / 2, K_{\mathrm{I}}^{\prime}$ can be calculated as $K_{\mathrm{I}}^{\prime}=K_{\mathrm{I}}+2 \tau_{0} \sqrt{\pi l}$.

Compared to stress intensity factor, energy release rate is more general to build the crack propagation criterion. Only $\sigma_{z z}\left(r, 0^{+}\right)$ and $u_{z}\left(r, 0^{+}\right)$are needed to calculate $G$ for mode-I crack problems $[16,17]$. Using Eqs. (28) and (30), one has

$$
G=\lim _{\Delta l / l \rightarrow 0^{+}} \frac{2^{1+e_{\mathrm{P}}+e_{\mathrm{T}}} \Gamma\left(1+e_{\mathrm{P}}\right) \Gamma\left(2+e_{\mathrm{T}}\right)}{\Gamma\left(3+e_{\mathrm{P}}+e_{\mathrm{T}}\right)} A_{\mathrm{P}} B_{\mathrm{T}}\left(\frac{\Delta l}{l}\right)^{1+e_{\mathrm{P}}+e_{\mathrm{T}}}
$$

in which $\Delta l$ is the distance that the crack propagates and $\Gamma(*)$ is the Gamma function. For $e_{\mathrm{P}}+e_{\mathrm{T}} \geq-1, G$ is finite and valid in the determination of crack growth; otherwise, $G$ is infinite. This criterion is looser than $e_{\mathrm{T}}>-1 / 2$, which is derived from stress intensity. A positive and infinite $G$ represents infinite energy being needed to fracture a material, and a negative and infinite $G$ represents an unphysical phenomenon that a spontaneous crack grows with releasing infinite strain energy from a material.

\section{The Toughness-Dominant Regime-Asymptotic Solution}

In the toughness-dominant regime, where the work done by fracturing the rock is much more than that done by overcoming the friction of the fluid flow, crack opening and pressure are characterized by $\sqrt{l-r}$ and $\ln (l-r)$, respectively [12]. According to Eq. (6), the shear stress is $O\left[(l-r)^{-1 / 2}\right]$, which results in a stress field of the same order as that induced by pressure. The stress intensity factor represents the amplitude of the stress field in the vicinity of the crack tip. As discussed in Sec. 3, it is rational to use $\sigma_{11}$ to calculate the stress intensity factor. Assuming that the mode-I fracture toughness is valid, one can use this stress intensity factor to build the crack propagation criterion. The criterion of crack propagation becomes, therefore

$$
\begin{aligned}
K_{\mathrm{I}}^{\prime}= & \frac{2}{\sqrt{\pi l(t)}} \int_{0}^{l(t)} \frac{p(\zeta, t) \zeta \mathrm{d} \zeta}{\sqrt{l^{2}(t)-\zeta^{2}}}+\sqrt{\frac{\pi}{l(t)}} \lim _{r \rightarrow l^{-}} \sqrt{l^{2}(t)-r^{2}} w(r, t) \\
& \times \frac{\partial p(r, t)}{\partial r} \geq K_{\mathrm{IC}}
\end{aligned}
$$

The fluid-driven crack problem is mathematically expressed with Eqs. (5), (6), (23), (33) and a certain initial condition such as the initial crack length $l_{0}$. Consequently, crack opening, crack length, 
and pressure are functions of $r, t, C^{\prime}, n, \mu, \nu, K_{\mathrm{IC}}, l_{0}$, and $q_{0}$. The normalization scheme is chosen to be

$$
r=l_{0} \tilde{l}(\tilde{t}) \tilde{r}, w(r, t)=\varepsilon l_{0} \tilde{w}(\tilde{r}, \tilde{t}), \quad p(r, t)=P \tilde{p}(\tilde{r}, \tilde{t}) \text { and } t=T \tilde{t}
$$

where

$$
\varepsilon=\frac{4 K_{\mathrm{IC}}}{\sqrt{\pi l_{0}} E^{\prime}}, \quad P=\frac{K_{\mathrm{IC}}}{2} \sqrt{\frac{\pi}{l_{0}}} \quad \text { and } \quad T=\frac{8 \sqrt{\pi} l_{0}^{5 / 2} K_{\mathrm{IC}}}{E^{\prime} q_{0}}
$$

Two dimensionless parameters are obtained as

$$
\tilde{C}=\frac{E^{2 n+1} q_{0}^{n} C^{\prime}}{2^{5 n+1} l_{0}^{2 n-1} K_{\mathrm{IC}}^{2 n+2}} \quad \text { and } \quad \tilde{Q}=\frac{(1-2 \nu) K_{\mathrm{IC}}}{2(1-\nu) E^{\prime}} \sqrt{\frac{\pi}{l_{0}}}
$$

which represent the ratio of viscous dissipation to the work of fracturing and the ratio of shear stress-caused crack opening to the pressure-caused crack opening, respectively. Using the normalization scheme, one obtains

$$
\begin{gathered}
\tilde{t}=\tilde{l}^{2}(\tilde{t}) \int_{0}^{1} \tilde{w}(\tilde{\zeta}, \tilde{t}) \tilde{\zeta} \mathrm{d} \tilde{\zeta} \\
\tilde{w}(\tilde{r}, \tilde{t})=\tilde{l}(\tilde{t}) \int_{0}^{1} p(\tilde{\zeta}, \tilde{t}) K_{\mathrm{P} 00}(\tilde{\zeta}, \tilde{r}) \mathrm{d} \tilde{\zeta}+\tilde{Q} \int_{\tilde{r}}^{1} \tilde{w}(\tilde{\zeta}, \tilde{t}) \frac{\partial \tilde{p}(\tilde{\zeta}, \tilde{t})}{\partial \tilde{\zeta}} \mathrm{d} \tilde{\zeta} \\
\frac{\partial \tilde{p}(\tilde{r}, \tilde{t})}{\partial \tilde{r}} \tilde{w}^{2 n+1}(\tilde{r}, \tilde{t}) \\
=-\tilde{C} \tilde{l}(\tilde{t})\left\{\frac{1}{\tilde{r}} \int_{\tilde{r}}^{1}\left[\tilde{l}(\tilde{t}) \frac{\partial \tilde{w}(\tilde{\zeta}, \tilde{t})}{\partial \tilde{t}}-\tilde{l}^{\prime}(\tilde{t}) \frac{\partial \tilde{w}(\tilde{\zeta}, \tilde{t})}{\partial \tilde{\zeta}}\right] \tilde{\zeta} \mathrm{d} \tilde{\zeta}\right\}^{n} \\
1=\sqrt{\tilde{l}(\tilde{t})} \int_{0}^{1} \frac{\tilde{\zeta} \tilde{p}(\tilde{\zeta}, \tilde{t})}{\sqrt{1-\tilde{\zeta}^{2}}} \mathrm{~d} \tilde{\zeta}+\frac{\pi \varepsilon}{2 \sqrt{\tilde{l}(\tilde{t})}} \lim _{\tilde{r} \rightarrow \tilde{l}} \sqrt{\tilde{l}^{2}(\tilde{t})-\tilde{r}^{2} \tilde{w}(\tilde{r}, \tilde{t}) \frac{\partial \tilde{p}(\tilde{r}, \tilde{t})}{\partial \tilde{r}}}
\end{gathered}
$$

with the initial condition being $\tilde{l}\left(\tilde{t}_{0}\right)=1$ in which $\tilde{t}_{0}$ is an unknown variable that is obtained by solving Eq. (37).

Perturbation theory is used to solve Eqs. (37)-(40), where the dependent variables are expanded with $\tilde{C}$ as

$$
\left\{\begin{array}{l}
\tilde{l}(\tilde{t})=\tilde{l}_{0}(\tilde{t})+\tilde{C} \tilde{l}_{1}(\tilde{t})+\tilde{C}^{2} \tilde{l}_{2}(\tilde{t})+\cdots \\
\tilde{w}(\tilde{r}, \tilde{t})=\tilde{w}_{0}(\tilde{r}, \tilde{t})+\tilde{C} \tilde{w}_{1}(\tilde{r}, \tilde{t})+\tilde{C}^{2} \tilde{w}_{2}(\tilde{r}, \tilde{t})+\cdots \\
\tilde{p}(\tilde{r}, \tilde{t})=\tilde{p}_{0}(\tilde{r}, \tilde{t})+\tilde{C} \tilde{p}_{1}(\tilde{r}, \tilde{t})+\tilde{C}^{2} \tilde{p}_{2}(\tilde{r}, \tilde{t})+\cdots
\end{array}\right.
$$

The first two orders of the asymptotic expansions are

$$
\tilde{l}_{0}(\tilde{t})=3^{\frac{2}{5}} \tilde{t}^{\frac{2}{5}}, \quad \tilde{w}_{0}\left(\tilde{x}_{1}, \tilde{t}\right)=3^{\frac{1}{5}} \tilde{t}^{\frac{1}{5}} \sqrt{1-\tilde{r}^{2}} \text { and } \tilde{p}_{0}\left(\tilde{x}_{1}, \tilde{t}\right)=3^{-\frac{1}{5} \tilde{t}^{-\frac{1}{5}}}
$$

and

$$
\left\{\begin{aligned}
\tilde{l}_{1}(\tilde{t})= & -\frac{34}{75}+\frac{17}{3^{1 / 5} 25} \tilde{Q} \tilde{t}^{\frac{1}{5}}-\frac{6 \pi}{25} \varepsilon \\
\tilde{w}_{1}(\tilde{r}, \tilde{t})= & \left(\frac{58}{3^{1 / 5} 75} \tilde{t}^{-\frac{1}{5}}+\frac{7}{3^{2 / 5} 50} \tilde{Q} \tilde{t}^{-\frac{2}{5}}+\frac{3^{4 / 5} 4 \pi}{25} \varepsilon \tilde{t}^{-\frac{1}{5}}\right) \sqrt{1-\tilde{r}^{2}} \\
& +\frac{3^{4 / 5} 2}{5} \tilde{t}^{-\frac{1}{5}} I(\tilde{r})-3^{-1 / 5} \tilde{t}^{-\frac{1}{5}} \tilde{r} \arccos \tilde{r}-3^{-2 / 5} \tilde{t}^{-\frac{2}{5}} \tilde{Q} \ln \frac{1+\sqrt{1-\tilde{r}^{2}}}{\tilde{r}} \\
\tilde{p}_{1}(\tilde{r}, \tilde{t})= & -\frac{17}{3^{4 / 5} 50} \tilde{Q} t^{-\frac{4}{5}}+\frac{3^{7 / 5} 2 \pi \varepsilon}{25} \tilde{t}^{-\frac{3}{5}}+\frac{3^{2 / 5}(32+75 \ln 2)}{225} \tilde{t}^{-\frac{3}{5}} \\
& -3^{2 / 5} \tilde{t}^{-\frac{3}{5}}\left[\frac{1}{3} \ln \tilde{r}-\frac{1}{5} \ln \left(1-\tilde{r}^{2}\right)\right]
\end{aligned}\right.
$$


(a)

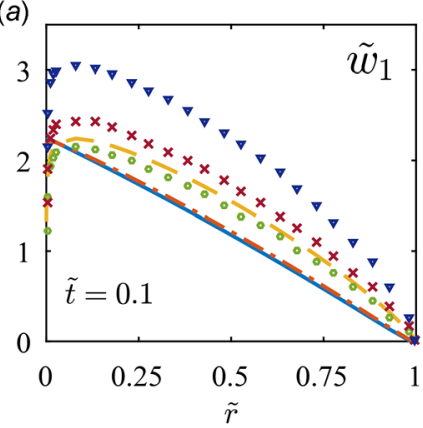

(b)

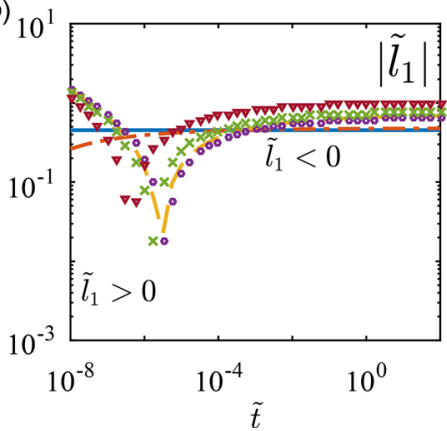

(c)

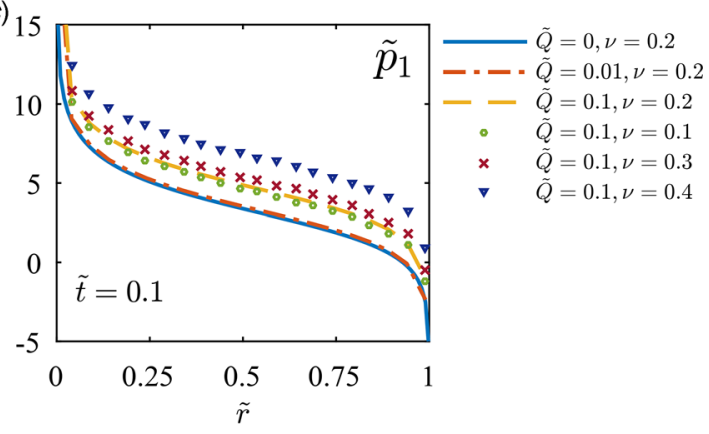

Fig. 3 First-order asymptotic expansion of crack opening, crack length, and pressure in the toughness-dominant regime

with

$$
I(\tilde{r})=\int_{\tilde{r}}^{1} \frac{\sqrt{1-\tilde{\zeta}^{2}} \arcsin \tilde{\zeta} \mathrm{d} \tilde{\zeta}}{\sqrt{\tilde{\zeta}^{2}-\tilde{r}^{2}}}
$$

in which there are two dimensionless parameters, $\tilde{Q}$ and $\varepsilon$, which are dependent for a known Poisson's ratio. For $\tilde{C}=0$, the asymptotic solution degenerates to the solution to the pressure-only model, which was obtained by Savitski and Detournay [12]. The zeroth-order asymptotic expansion represents a penny-shaped crack driven by an inviscid fluid and shows no new information other than the toughness scaling [19]. The first-order asymptotic expansion takes effect for a relatively larger value of $\tilde{C}$, but the singularities always exist.

The first-order asymptotic expansion is nontrivial due to the existence of fluid viscosity. A larger $\tilde{Q}$ leads to a smaller crack opening and a larger pressure at a same point near the crack center, and the effect of a constant $\tilde{Q}$ increases with the increase of Poisson's ratio. It is evident that the first-order asymptotic expansions of crack opening and pressure are singular. As shown in Figs. 3(a) and 3(c), both crack opening and pressure are singular at the wellbore for $\tilde{Q}$ being non-zero. The singular pressure has been discussed in the toughness-dominant regime [11-13], and the singularities can be removed by considering the diameter of the wellbore and the fluid-lag zone near the crack tip. The singular crack opening, which is limited in the vicinity of the crack center and the crack tip, is due to the existence of shear stress. Because the singularity of shear stress is stronger than pressure according to Eq. (6), and a singular crack opening is obtained from the second term on the right-hand side of Eq. (38). Even though the singularities are unphysical and removable, they indicate potential instabilities and failures to release the concentration of stress.

According to Fig. 3(b), the existence of shear stress leads to a positive first-order expansion of crack length at a small time and a negative one at a large time, and the transition is smooth over time. A plausible reason is the competition between shear stress and stress intensity factor. In the first stage, shear stress decreases the crack opening leading to a higher pressure drop and a lower stress intensity factor; and in the second stage, a higher wellbore pressure is rebuilt to fracturing the rocks leading to a larger crack opening and a small value of shear stress. At a small time, the effect of shear stress is dominant. A smaller crack opening is therefore obtained, and a longer crack is fractured because of the fluid mass conservation. At a large time, the effect of stress intensity factor becomes dominant. A larger crack opening is obtained and the first-order expansion of crack length is negative. As shown in Fig. 4, the first-order expansion of crack opening is negative at $\tilde{t}=10^{-6}$ and gradually becomes positive, and the negative crack opening at the wellbore becomes increasingly negligible with time. Even though the small time, e.g., $\tilde{t}=10^{-6}$, is not easy to be obtained in experiments, it is meaningful in the

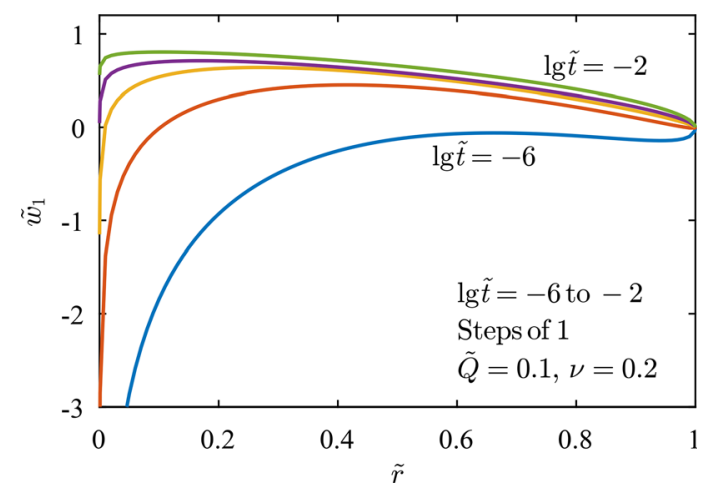

Fig. 4 Time evolution of the first-order asymptotic expansion of crack opening

theoretical analysis due to the transition between the dominant effects of shear stress and fracture toughness.

\section{The Viscosity-Dominant Regime}

For a full-stress model in the viscosity-dominant regime, where the work done by fracturing the rock is much less than that done by overcoming the friction of the fluid flow, the crack may propagate unstably. The crack opening and pressure near the crack tip are characterized by $(l-r)^{2 / 3}$ and $-(l-r)^{-1 / 3}$, respectively, by using a pressure-only model [12]. Shear stress is obtained from Eq. (6) as $-O\left[(l-r)^{-2 / 3}\right]$. Consequently, there is an unconvergent stress intensity factor and a negatively finite strain energy release rate according to Eqs. (31) and (32). However, crack arrest has not been reported in the past visible experiments [21,23-25]. There should be a criterion for the shear stress-induced crack arrest.

Here, it is demonstrated, by using scaling analysis, that crack arrest disappears at a large dimensionless time due to the decreasing effect of shear stress. In a scaling analysis, it is convenient to neglect the other relevant physical effects for one physical effect being dominant [26]. The physical quantities become time independent after the self-similar transformation. For a penny-shaped crack, a set of simplified transforms

$$
\begin{aligned}
l(t) & =L(t), \quad w(r, t)=W(t) \tilde{w}(\tilde{r}), \quad p(r, t)=\Sigma(t) \tilde{p}(\tilde{r}) \quad \text { and } \\
r & =L(t) \tilde{r}
\end{aligned}
$$

are used for the scaling analysis. In Eqs. (45), $L(t), W(t)$, and $\Sigma(t)$ can be either exponential or power functions $[1,19]$, which differ from the boundary condition of flow rate. Using the power functions $[12,13,15,18]$, one obtains from the Eqs. (5), (6), (23), and (33) that 


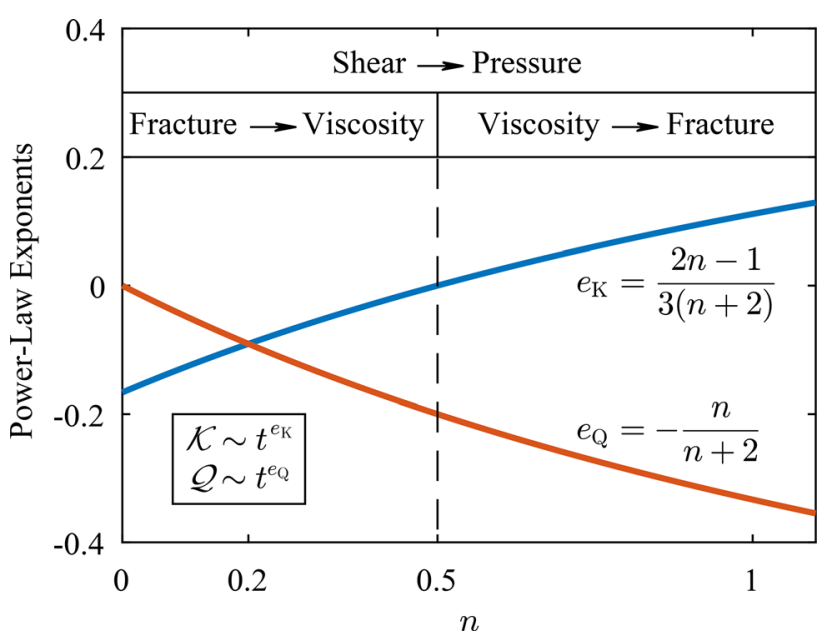

Fig. 5 Transition of the dominant regimes with respect to the flow behavior index

$\mathcal{V}=\frac{q_{0} t}{2 \pi L^{2}(t) W(t)}, \quad \mathcal{P}=\frac{8 \Sigma(t) L(t)}{\pi E^{\prime} W(t)}, \quad \mathcal{M}=\frac{C^{\prime} L^{n+1}(t) W^{\prime n}(t)}{\sum(t) W^{2 n+1}(t)}$

$\mathcal{K}=\frac{K_{\mathrm{IC}}}{2 \Sigma(t)} \sqrt{\frac{\pi}{L(t)}} \quad$ and $\quad \mathcal{Q}=\frac{\pi(1-2 \nu) W(t)}{8(1-\nu) L(t)}$

where $\mathcal{V}$ is from the mass conservation; $\mathcal{P}$ is from the boundary integral of crack opening under pressure; $\mathcal{M}$ is from the lubrication theory; $\mathcal{K}$ is from the crack propagation criterion; and $\mathcal{Q}$ is from the boundary integral of crack opening under shear stress. Following Refs. [12], [18], and [19] $\mathcal{V}=\mathcal{P}=\mathcal{M}=1$ is used to ensure the mass conservation and globally positive crack opening in the viscosity-dominant regime. Consequently, there are

$$
\begin{aligned}
L(t) & =\left(\frac{q_{0}^{n+2} E^{\prime}}{C^{\prime}}\right)^{\frac{1}{3(n+2)}} t^{\frac{2(n+1)}{6+3 n}} \\
W(t) & =\left(\frac{q_{0}^{n+2} C^{\prime 2}}{E^{\prime 2}}\right)^{\frac{1}{3(n+2)}} t^{\frac{2-n}{6+3 n}} \\
\Sigma(t) & =\left(C^{\prime} E^{\prime n+1}\right)^{\frac{1}{n+2}} t^{-\frac{n}{n+2}}
\end{aligned}
$$

with two dimensionless groups

$$
\mathcal{K}=\frac{K_{\mathrm{IC}}}{\left(q_{0}^{n+2} C^{\prime 5} E^{\prime 6 n+7}\right)^{\frac{1}{6(n+2)}}} t^{\frac{2 n-1}{3(n+2)}} \quad \text { and } \quad \mathcal{Q}=\frac{1-2 \nu}{1-\nu}\left(\frac{C^{\prime}}{E^{\prime}}\right)^{\frac{1}{n+2}} t^{-\frac{n}{n+2}}
$$

which represent the ratio of the work of fracturing rocks to viscous dissipation and the ratio of shear stress-caused crack opening to the pressure-caused crack opening, respectively. The powerlaw exponents $e_{\mathrm{K}}$ and $e_{\mathrm{Q}}$ of $\mathcal{Q} \sim t^{e_{\mathrm{Q}}}$ and $\mathcal{K} \sim t^{e_{\mathrm{K}}}$ are shown in Fig. 5 for various $n$. The temporal evolution of the two dimensionless groups gives the dominant-regime transitions between shear stress and pressure, and between viscosity and fracture toughness. It is evident that for a fracturing fluid with power-law rheology, the shear stress dominates the problem either for the flow consistency index being very large or for the time being small so that $\mathcal{Q}$ is sufficiently large. The two conditions are not possible to achieve in the indoor experiments, where well-prepared rocks or glasses were used. However, the fluid channel constructed by fracturing a laminated rock consists of corners, throats, and other secondary structures, which can lead to very high hydraulic resistance, and the effect of shear stress is much underestimated in this analysis.

The near-crack-tip stress field in the rock can be obtained from the local properties of pressure and shear stress. Similar to the problem of a uniform normal stress acting on the crack surfaces, a stress component can be reduced to two separate factors, a radial component and an angular component. The former is determined
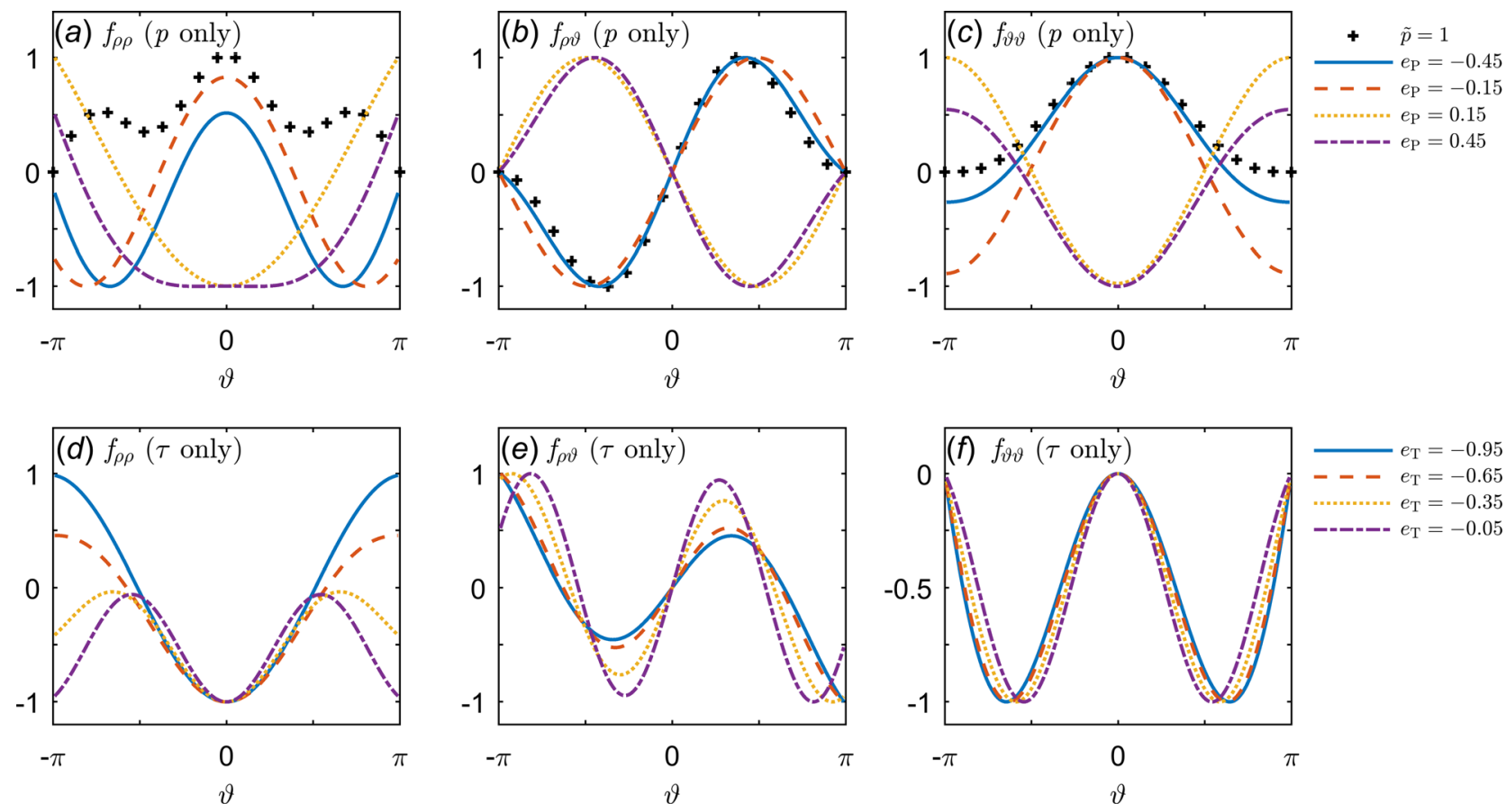

Fig. 6 Angular distribution of normalized stress components around the crack tip. The normalized distance from the crack tip is $\tilde{\rho}=10^{-4}$. 
by the radial coordinate and boundary conditions, and the latter is determined by the power-law exponent of either pressure or shear stress. The angular component is the normalized angular distribution of the stress component, which helps in the determination of crack deflection. A polar coordinate system is introduced at the crack tip as

$$
\rho=\sqrt{(r-l)^{2}+z^{2}}, \quad \text { and } \quad \vartheta=\operatorname{Arg}(r-l+\mathrm{i} z)
$$

where Arg represents the argument of a complex number, and $\rho$ is normalized by $l$. By assuming power-law pressure and shear stress distributions in Eq. (27), the stress components in the rock are calculated with Eqs. (15)-(17). The normalized angular distributions of stress components near the crack tip are plotted in Fig. 6 for normal stress-only and shear stress-only problems. $f_{\rho \rho}, f_{\rho \vartheta}$, and $f_{\vartheta \vartheta}$ represent the normalized $\sigma_{\rho \rho}, \sigma_{\rho \vartheta}$, and $\sigma_{\vartheta \vartheta}$, respectively. It is evident that $\sigma_{\rho \rho}$ and $\sigma_{\vartheta \vartheta}$ are in tensile state for $\tilde{p}=1$, but there are both tensile and compressive states for $\sigma_{\rho \rho}$ and $\sigma_{\vartheta \vartheta}$ around the crack tip for $e_{\mathrm{P}}<0$. The maxima of $\sigma_{\rho \rho}$ and $\sigma_{\vartheta \vartheta}$ are at $\vartheta=0$, and the minima are near the crack surfaces. And for $e_{\mathrm{P}}>0$, negative stress components are obtained and in accord with Sec. 4. For shear stress-only problems, $\sigma_{\vartheta \vartheta}$ is negative except for $\vartheta=0$ and $\pm \pi$, where $\sigma_{\vartheta \vartheta}=0$. This means that the material is in compressive state and possible to be fractured either straightly or perpendicularly to the crack surface. Note that $\sigma_{\rho \rho}$ is non-zero and minimum at $\vartheta=0$, where $\sigma_{\vartheta \vartheta}$ is zero. Consequently, the use of $\sigma_{\vartheta \vartheta}(\rho, 0)$ does not reveal the asymptotic property of the stress field. The analysis of the near-crack-tip stress field is based on the singularities of pressure and shear stress. Either a fluid-lag zone or a two-dimensional flow assumption will eliminate the singularities $[10,19,27]$. Even though the fluid-lag zone, or the region of two-dimensional flow, with a negligible size influences the distribution of the stress field slightly because the stress singularities are integrable. A global solution to the fluid-driven crack problem with a fluid-lag zone is still needed by using the full-stress model.

\section{Conclusions}

A full-stress model is proposed to analyze the combined effect of pressure and shear stress acting on the crack surfaces for a penny-shaped fluid-driven crack. The boundary integral equations of displacement and stress in the full linear elastic space with crack are derived by integral transform method. An asymptotic solution is derived in the toughness-dominant regime.

A negative shear stress (i.e., $\sigma_{r z}\left(r, 0^{+}\right)<0$ and $\sigma_{r z}\left(r, 0^{-}\right)>0$ ) leads to the decrease of the normal displacement and stress and tends to inhibit the crack. For cracks under power-law loads, the exponents of the power-law shear stress may change the asymptotic properties of the solution, which results in the change of the order of the stress singularity at the crack tip. For problems with nonzero shear stress acting on the crack surfaces, $\sigma_{r r}\left(r, 0^{+}\right)$, rather than $\sigma_{z z}\left(r, 0^{+}\right)$, should be used to calculate the stress intensity factor, because the latter is independent of the shear stress. The crack propagation criterion of stress intensity factor is conditionally valid for power-law loads. The strain energy release rate is proved to be more universal than the stress intensity factor. There are dominant-regime transitions between shear stress and pressure, and between fracture and viscosity. The transitions are dependent on the flow behavior index. Unstable crack propagation occurs in the shear stress-dominant regime. The effect of shear stress decreases with time. Shear stress leads to a smaller crack opening, a longer crack and a slightly larger wellbore pressure. Singularities of stress components and crack opening are found at the crack center, which reveals potential instabilities and failures at the wellbore.

This study gives a theoretical analysis on the combined effect of pressure and shear stress in hydraulic fracturing, and will be useful for future investigations of the fluid-solid interaction problems.

\section{Funding Data}

- National Natural Science Foundation of China (Grant Nos. 11372313, 11611130019, and U1562105).

- Chinese Academy of Sciences (CAS) through CAS Interdisciplinary Innovation Team Project, the CAS Key Research Program of Frontier Sciences (Grant No. QYZDJ-SSW449JSC019).

- CAS Strategic Priority Research Program (Grant No. XDB22040401).

\section{Nomenclature}

$A_{\mathrm{P}}, A_{\mathrm{T}}, B_{\mathrm{P}}, B_{\mathrm{T}}=$ coefficients of the asymptotic solution, subscripts "K", "P" and "T" represent stress intensity factor, normal and shear stress, respectively

$\tilde{C}=$ dimensionless flow consistency index

$E^{\prime}=2 \mu /(1-\nu)$

$f_{\rho \rho}, f_{\rho \vartheta}, f_{\vartheta \vartheta}=$ normalized stress components of the polar coordinate system at crack tip

$G=$ energy release rate

$I_{\mathrm{P} i j}, I_{\mathrm{T} i j}=$ functions of $r$ and $z$, subscripts "P" and "T" represent the pressure and shear stress

$\mathcal{I}_{i j}=$ infinite integral of the inverse Hankel transform

$K_{\mathrm{I}}=$ mode I stress intensity factor calculated with $\sigma_{z z}$

$K_{\mathrm{I}}^{\prime}=$ mode I stress intensity factor calculated with $\sigma_{r r}$

$K_{\mathrm{IC}}=$ fracture toughness

$K_{\mathrm{P} i j}, K_{\mathrm{T} i j}=$ kernel functions related to $I_{\mathrm{P} i j}$ and $I_{\mathrm{T} i j}$, respectively

$\mathcal{K}=$ time-dependent dimensionless group of $\tilde{K}_{\text {IC }}^{\prime}$

$l=$ crack length

$p=$ distribution of pressure

$\tilde{Q}=$ dimensionless parameter representing the ratio of shear-stress-caused crack opening to the pressure-caused crack opening

$\mathcal{Q}=$ time-dependent dimensionless group of $\tilde{Q}$

$u_{r}, u_{z}=$ displacement components

$w=$ crack opening (width of the fluid channel)

$\gamma, \gamma=$ strain tensor and its trace

$\Gamma=$ Gamma function

$\varepsilon=$ dimensionless parameter

$\mu, \nu=$ Shear modulus and Poisson's ratio

$\boldsymbol{\sigma}, \sigma_{r r}, \sigma_{r z}, \sigma_{z z}=$ stress tensor and its components

$\sigma_{\rho \rho}, \sigma_{\rho \vartheta}, \sigma_{\vartheta \vartheta}=$ stress components of the polar coordinate system at crack tip

$\sigma_{\infty}=$ uniform stress at infinity

$\tau=$ distribution of shear stress

The symbol " " above a variable represents that the variable is dimensionless

\section{Appendix A: Derivation}

Here, Eqs. (1) and (2) are derived. The viscous flow in the very thin channel between the crack surfaces is approximately one-dimensional. Pressure is, therefore, the function of coordinate $r$ and time only. The balance of force in the fracturing fluid is

$$
\frac{\partial p(r, t)}{\partial r}=\frac{\partial \tau(r, z, t)}{\partial z}
$$

Using the Ostwald-de Waele relationship, $\tau=C \operatorname{sgn}\left\{\partial v_{z} / \partial z\right\}\left|\partial v_{z} / \partial z\right|^{n}$, Eq. (A1) is converted into

$$
\frac{\partial p(r, t)}{\partial r}=C \frac{\partial}{\partial z}\left[\operatorname{sgn}\left\{\frac{\partial v_{z}(r, z, t)}{\partial z}\right\}\left|\frac{\partial v_{z}(r, z, t)}{\partial z}\right|^{n}\right]
$$


The mass conservation holds due to no sink and source inside the channel. Consequently, there is

$$
\frac{1}{r} \frac{\partial}{\partial r}\left[r v_{r}(r, z, t)\right]+\frac{\partial v_{z}(r, z, t)}{\partial z}=0
$$

Equations (A2) and (A3) are the governing equations of the viscous fluid flow. The integration of Eqs. (A2) and (A3) requires velocity boundary conditions. Using the symmetry of the geometry, one has

$$
\begin{gathered}
v_{r}\left(r,-\frac{w(r, t)}{2}, t\right)=v_{r}\left(r, \frac{w(r, t)}{2}, t\right)=\frac{\partial v_{r}(r, 0, t)}{\partial z}=0 \\
v_{z}\left(r, \frac{w(r, t)}{2}, t\right)=-v_{z}\left(r,-\frac{w(r, t)}{2}, t\right)=\frac{1}{2} \frac{\partial w(r, t)}{\partial t}
\end{gathered}
$$

Integrating Eq. (A2) twice across the fluid channel with Eq. (A4), and Eq. (A3) across the fluid channel with Eq. (A5) gives

$$
\begin{aligned}
& v_{r}(r, z, t)= \frac{n}{n+1}\left(-\frac{1}{C} \operatorname{sgn}\left\{\frac{\partial p(r, t)}{\partial r}\right\}\left|\frac{\partial p(r, t)}{\partial r}\right|\right)^{\frac{1}{n}} \\
& \times\left\{\left[\frac{w(r, t)}{2}\right]^{1+\frac{1}{n}}-z^{1+\frac{1}{n}}\right\} \\
& \frac{1}{r} \frac{\partial}{\partial r}\left[r \int_{-w(r, t) / 2}^{w(r, t) / 2} v_{r}(r, z, t) \mathrm{d} z\right]+\frac{\partial w(r, t)}{\partial t}=0
\end{aligned}
$$

The flow rate across a section of the fluid channel, i.e., a cylindrical surface with its radius being $r$ and its height being $w(r, t)$, is defined by

$$
q(r, t)=2 \pi r \int_{-w(r, t) / 2}^{w(r, t) / 2} v_{r}(r, z, t) \mathrm{d} z
$$

Thus, Eqs. (A6) and (A7) are rewritten as

$$
q(r, t)=-\operatorname{sgn}\left\{\frac{\partial p(r, t)}{\partial r}\right\} \frac{4 n \pi r}{C^{1 / n} 2^{(2 n+1) / n}(2 n+1)}\left|\frac{\partial p(r, t)}{\partial r} w^{2 n+1}(r, t)\right|^{\frac{1}{n}}
$$

$$
\frac{\partial w(r, t)}{\partial t}+\frac{1}{2 \pi r} \frac{\partial q(r, t)}{\partial r}=0
$$

\section{Appendix B: Single Integral-Based Solution for $z=0$}

The iterated integral-based solution can be reduced to single integral based solution by changing the order of integration. There are, for $0<r<l$

$$
\begin{aligned}
u_{r}\left(r, 0^{+}\right)= & -\frac{1-2 \nu}{2 \mu r} \int_{0}^{r} \zeta p(\zeta) \mathrm{d} \zeta+\frac{2(1-\nu)}{\pi \mu} \int_{0}^{r} K_{\mathrm{T} 10}(\zeta ; r) \tau(\zeta) \mathrm{d} \zeta \\
u_{z}\left(r, 0^{+}\right)= & \frac{2(1-\nu)}{\pi \mu} \int_{0}^{l} K_{\mathrm{P} 00}(\zeta ; r) p(s) \mathrm{d} s+\frac{1-2 \nu}{2 \mu} \int_{r}^{l} \tau(\zeta) \mathrm{d} \zeta(\mathrm{B} 2) \\
\sigma_{r r}\left(r, 0^{+}\right)= & -p(r)+\frac{1-2 \nu}{r^{2}} \int_{0}^{r} \zeta p(\zeta) \mathrm{d} \zeta \\
& +\frac{4}{\pi r} \int_{0}^{1} K_{\mathrm{T} 11}(\zeta ; r) \tau(\zeta) \mathrm{d} \zeta-\frac{4(1-\nu)}{\pi r} \int_{0}^{l} K_{\mathrm{T} 10}(\zeta ; r) \tau(\zeta) \mathrm{d} \zeta
\end{aligned}
$$

and for $r>l$

$$
\begin{aligned}
u_{r}(r, 0)= & \frac{1-2 \nu}{\pi \mu r} \int_{0}^{l}\left(\arctan \sqrt{\frac{r^{2}-l^{2}}{l^{2}-\zeta^{2}}}-\frac{\pi}{2}\right) \zeta p(\zeta) \mathrm{d} \zeta \\
& +\frac{2(1-\nu)}{\pi} \int_{0}^{r} K_{\mathrm{T} 10}(\zeta ; r) \tau(\zeta) \mathrm{d} \zeta
\end{aligned}
$$

$\sigma_{r r}(r, 0)=\frac{2}{\pi} \frac{1}{\sqrt{r^{2}-l^{2}}} \int_{0}^{l} \frac{\sqrt{l^{2}-\zeta^{2}}}{r^{2}-\zeta^{2}} \zeta p(\zeta) \mathrm{d} \zeta$

$$
\begin{aligned}
& +\frac{2(1-2 \nu)}{\pi r^{2}} \int_{0}^{l}\left(\frac{\pi}{2}-\arctan \sqrt{\frac{r^{2}-l^{2}}{l^{2}-\zeta^{2}}}\right) \zeta p(\zeta) \mathrm{d} \zeta \\
& +\frac{4}{\pi r} \int_{0}^{1} K_{\mathrm{T} 11}(\zeta ; r) \tau(\zeta) \mathrm{d} \zeta-\frac{4(1-\nu)}{\pi r} \int_{0}^{l} K_{\mathrm{T} 10}(\zeta ; r) \tau(\zeta) \mathrm{d} \zeta
\end{aligned}
$$

$$
\sigma_{z z}(r, 0)=\frac{2}{\pi} \frac{1}{\sqrt{r^{2}-l^{2}}} \int_{0}^{l} \frac{\sqrt{l^{2}-\zeta^{2}}}{r^{2}-\zeta^{2}} \zeta p(\zeta) \mathrm{d} \zeta
$$

where the kernel functions

$$
\begin{aligned}
K_{\mathrm{P} 00}(\zeta ; r)= \begin{cases}\frac{\zeta}{r} \mathbb{F}\left(\arcsin \sqrt{\frac{l^{2}-r^{2}}{l^{2}-\zeta^{2}}}, \frac{\zeta^{2}}{r^{2}}\right), & \zeta \in(0, r) \\
\mathbb{F}\left(\arcsin \sqrt{\frac{l^{2}-\zeta^{2}}{l^{2}-r^{2}}}, \frac{r^{2}}{\zeta^{2}}\right), & \zeta \in(r, l)\end{cases} \\
K_{\mathrm{T} 10}(\zeta ; r)= \begin{cases}\mathbb{E}\left(\frac{\zeta^{2}}{r^{2}}\right)-\mathbb{K}\left(\frac{\zeta^{2}}{r^{2}}\right), & \zeta \in(0, r) \\
\frac{\zeta}{r}\left[\mathbb{E}\left(\frac{r^{2}}{\zeta^{2}}\right)-\mathbb{K}\left(\frac{r^{2}}{\zeta^{2}}\right)\right], & \zeta \in(r, l)\end{cases}
\end{aligned}
$$

$$
K_{\mathrm{T} 11}(\zeta ; r)= \begin{cases}\frac{r^{2}}{r^{2}-\zeta^{2}} \mathbb{E}\left(\frac{\zeta^{2}}{r^{2}}\right)-\mathbb{K}\left(\frac{\zeta^{2}}{r^{2}}\right), & \zeta \in(0, r) \\ \frac{r \zeta}{r^{2}-\zeta^{2}} \mathbb{E}\left(\frac{r^{2}}{\zeta^{2}}\right), & \zeta \in(r, l)\end{cases}
$$

are the finite integrals obtained by changing the order of $I_{\mathrm{P} i j}$ and $I_{\mathrm{T} i j} . \mathbb{F}(*, *), \mathbb{E}(*)$, and $\mathbb{K}(*)$ are the incomplete elliptic integral of the first kind, the complete elliptic integral of the first kind and the incomplete elliptic integral of the second kind, respectively. Note that stress singularities may exist at the crack tip and crack center. Thus, the solutions at the two points are obtained with, for displacement, $r \rightarrow 0^{+}$and $r \rightarrow l^{-}$, and for stress, $r \rightarrow 0^{+}$and $r \rightarrow l^{+}$.

\section{References}

[1] Spence, D. A., and Sharp, P., 1985, "Self-Similar Solutions for Elastohydrodynamic Cavity Flow,” Proc. R. Soc. London, Ser. A, 400(1819), pp. 289-313.

[2] Tsai, V. C., and Rice, J. R., 2010, “A Model for Turbulent Hydraulic Fracture and Application to Crack Propagation at Glacier Beds," J. Geophys. Res. Earth Surf., 115(F3), p. F03007.

[3] Lister, J. R., and Ross, C. K., 1991, "Fluid-Mechanical Models of Crack Propagation and Their Application to Magma Transport in Dykes," J. Geophys. Res. Solid Earth, 96(6), pp. 10049-10077.

[4] Yang, F. Q., and Zhao, Y. P., 2016, "The Effect of a Capillary Bridge on the Crack Opening of a Penny Crack," Soft Matter, 12(5), pp. 1586-1592.

[5] Verdon, J. P., Kendall, J. M., Stork, A. L., Chadwick, R. A., White, D. J., and Bissell, R. C., 2013, "Comparison of Geomechanical Deformation Induced by Megatonne-Scale $\mathrm{CO}_{2}$ Storage at Sleipner, Weyburn, and in Salah,” Proc. Natl. Acad. Sci. U. S. A., 110(30), pp. 2762-2771.

[6] Vasco, D., Rucci, A., Ferretti, A., Novali, F., Bissell, R., Ringrose, P., Mathieson, A., and Wright, I., 2010, "Satellite-Based Measurements of Surface 
Deformation Reveal Fluid Flow Associated With the Geological Storage of Carbon Dioxide," Geophys. Res. Lett., 37(3), p. L03303.

[7] Abe, H., Mura, T., and Keer, L. M., 1976, "Growth-Rate of a Penny-Shaped Crack in Hydraulic Fracturing of Rocks," J. Geophys. Res., 81(29), pp. $5335-5340$.

[8] Dong, X., Zhang, G., Gao, D., and Duan, Z., 2017, “Toughness-Dominated Hydraulic Fracture in Permeable Rocks," ASME J. Appl. Mech., 84(7), p. 071001.

[9] Mishuris, G., Wrobel, M., and Linkov, A., 2012, "On Modeling Hydraulic Fracture in Proper Variables: Stiffness, Accuracy, Sensitivity," Int. J. Eng. Sci., 61, pp. 10-23.

[10] Shen, W. H., and Zhao, Y. P., 2017, "Quasi-Static Crack Growth Under Symmetrical Loads in Hydraulic Fracturing," ASME J. Appl. Mech., 84(8), p. 081009.

[11] Geertsma, J., and de Klerk, F., 1969, "A Rapid Method of Predicting Width and Extent of Hydraulically Induced Fractures,” J. Pet. Technol., 21(12), pp. 1571-1581.

[12] Savitski, A. A., and Detournay, E., 2002, "Propagation of a Penny-Shaped Fluid-Driven Fracture in an Impermeable Rock: Asymptotic Solutions,” Int. J. Solids Struct., 39(26), pp. 6311-6337.

[13] Bunger, A. P., and Detournay, E., 2007, "Early-Time Solution for a Radial Hydraulic Fracture," J. Eng. Mech., 133(5), pp. 534-540.

[14] Garagash, D. I., and Detournay, E., 2000, "The Tip Region of a Fluid-Driven Fracture in an Elastic Medium," ASME J. Appl. Mech., 67(1), pp. 183-192.

[15] Garagash, D. I., Detournay, E., and Adachi, J. I., 2011, "Multiscale Tip Asymptotics in Hydraulic Fracture With Leak-Off," J. Fluid Mech., 669, pp. 260-297.

[16] Lawn, B., 1993, Fracture of Brittle Solids, Cambridge University Press, Cambridge, UK.
[17] Zhao, Y. P., 2016, Modern Continuum Mechanics, Science Press, Beijing, China (in Chinese)

[18] Detournay, E., 2004, "Propagation Regimes of Fluid-Driven Fractures in Impermeable Rocks," Int. J. Geomech., 4(1), pp. 35-45.

[19] Detournay, E., 2016, "Mechanics of Hydraulic Fractures," Annu. Rev. Fluid Mech., 48, pp. 311-339.

[20] Sneddon, I. N., and Lowengrub, M., 1969, Crack Problems in the Classical Theory of Elasticity, Wiley, New York.

[22] Yang, F., 1998, "Indentation of an Incompressible Elastic Film," Mech. Mater. 30(4), pp. 275-286.

[21] Bunger, A. P., and Detournay, E., 2008, "Experimental Validation of the Tip Asymptotics for a Fluid-Driven Crack," J. Mech. Phys. Solids, 56(11), pp. 3101-3115.

[23] Lai, C. Y., Zheng, Z., Dressaire, E., Ramon, G. Z., Huppert, H. E., and Stone, H. A., 2016, "Elastic Relaxation of Fluid-Driven Cracks and the Resulting Backflow," Phys. Rev. Lett., 117(26), p. 268001.

[24] Lai, C. Y., Zheng, Z., Dressaire, E., Wexler, J. S., and Stone, H. A., 2015, "Experimental Study on Penny-Shaped Fluid-Driven Cracks in an Elastic Matrix," Proc. R. Soc. A., 471(2182), p. 20150255.

[25] Lecampion, B. Desroches, J., Jeffrey, R. G., and Bunger, A. P. 2017, "Experiments Versus Theory for the Initiation and Propagation of Radial Hydraulic Fractures in Low-Permeability Materials," J. Geophys. Res: Solid Earth, 122(2), pp. 1239-1263.

[26] Zhao, Y. P., 2014, Nano and Mesoscopic Mechanics, Science Press, Beijing, China (in Chinese)

[27] Bui, H. D., 1977, "An Integral Equations Method for Solving the Problem of a Plane Crack of Arbitrary Shape," J. Mech. Phys. Solids, 25(1), pp. 29-39. 